\title{
Sobre algoritmos y eficiencias: fijación de precios y empresas de redes de transporte*
}

\section{Algorithms and Efficiency: Price-Fixing in Digital Transportation Networks}

Carlos Ignacio Arboleda Suárez ${ }^{\text {a }}$

Dentons Cárdenas \& Cárdenas, Colombia

carlos.arboleda@dentons.com

ORCID: https://orcid.org/0000-0003-0557-2772
DOI: https://doi.org/10.11144/Javeriana.vj69.saef

Recibido: 25 Octubre 2019

Aceptado: 08 Noviembre 2019

Publicado: 15 Agosto 2020

\section{Resumen:}

El sector de la movilidad urbana ha sido uno de los más afectados por la creciente utilización de aplicaciones móviles. Éstas han generado una reducción de costos de entrada y de transacción tan importantes que los mercados han crecido de forma asombrosa. Sin embargo, y como suele ser el caso, las normas no han evolucionado tan rápidamente. Las plataformas digitales suponen un reto en el ordenamiento jurídico, el cual no ha sido ajeno a nuestra concepción de libre competencia. Por ello, es importante entender los impactos económicos -negativos y positivos- que traen estas tecnologías de movilidad, entender su modelo de negocio, proceso de formación de precios y transacciones para, en últimas, evitar regularlos de manera equivocada con categorías legales que ya no se acoplan a la realidad.

Palabras clave: libre competencia, fijación de precios, movilidad, aplicaciones.

\section{Abstract:}

Urban transportation has been one of the economic sectors that has been impacted the most by mobile apps. These have reduced entry and transaction costs of such magnitude that markets have flourished. However, and as is often the case, regulation has not evolved as rapidly. These digital platforms create a challenge to regulation and have impacted our notion of free competition. Thus, it is important to understand the economic consequences -negative and positive- that these technologies create, better grasp their business model, price formation and transaction processes, to ultimately, avoid regulating them inappropriately via regulatory boxes that no longer fit reality.

Keywords: competition, price-fixing, transportation, apps.

\section{Acuerdos de fijación de precios en Colombia}

\section{Generalidades}

El numeral 1 del artículo 47 del Decreto 2153 de 1992 considera como contrario a la libre competencia cualquier acuerdo que tenga "por objeto o como efecto la fijación directa o indirecta de precios" ${ }^{1}$. Respecto de esta conducta, ha indicado la Superintendencia de Industria y Comercio (“SIC"):

[E]l sistema de libre competencia se traduce en garantía a la libertad de entrada y salida a los mercados, la libertad para que cada agente del mercado ofrezca el precio por sus productos o servicios que de forma independiente defina con base en su estructura de costos y las condiciones del mercado, que ofrezca las calidades y cantidades de productos que desee, y que los consumidores elijan libremente qué comprar. Bajo estas condiciones, los precios de mercado dan una señal a los consumidores sobre los niveles de oferta de un producto en el mercado y asigna con eficiencia los recursos disponibles.

(...)

Estas condiciones del sistema de libre competencia son benéficas porque contribuyen a un mejor desarrollo de la economía y tutelan de manera efectiva los intereses de todos los agentes del mercado y del Estado mismo. De acuerdo con lo anterior, las conductas que coarten la libre competencia e impidan la libre formación del precio, se consideran ilegales por violar las normas de protección de la libre competencia y se denominan anticompetitivas o restrictivas de la competencia. Entre estas

Notas de autor

a Autor de correspondencia. Correo electrónico: carlos.arboleda@dentons.com 
conductas, los carteles empresariales se distinguen por ser la conducta más dañina para los consumidores y la economía en general y es considerada como la conducta más reprochable en que puede incurrir un empresario. ${ }^{2}$

De acuerdo con el tenor literal de la norma antes transcrita, es sancionable tanto el objeto como el efecto de la conducta. El primero se refiere a la potencialidad de la conducta para causar un daño en el mercado; el segundo, al resultado de la actividad ${ }^{3}$. Además, en cuanto al efecto, la SIC ha considerado que el mismo no depende de la intención con la que actúen los agentes, en el sentido de ser sancionable independientemente de que los participantes del acuerdo hayan buscado dicho resultado ${ }^{4}$.

Con respecto a los precios como tal, la SIC ha indicado que la ley "en ningún momento establece que los precios del acuerdo deban ser perjudiciales o nocivos para el mercado, pues la ley asume que el solo hecho de que se elimine la variedad de precios ya supone un menoscabo para el consumidor" 5 .

Por otra parte, la norma cubre tanto la fijación directa como la indirecta de los precios. Según la SIC, “[1]os acuerdos entre competidores que se realizan con el fin de fijar precios, ocurren bien a través de la manipulación directa de la variable precio -fijación directa- o de cualquier otra que pueda influenciarla -fijación indirecta-" 6 . Entonces, la fijación de precios no sólo cubre el escenario en el que los agentes del mercado acuerdan un valor numérico, sino que, en palabras de la autoridad, incluye:

[C]ualquier otra forma, tales como porcentajes máximos o mínimos de descuento, rangos de fluctuación entre dos sumas, techo, piso, adopción de referenciales únicos o, en general cualquier mecanismo, limitación o condicionamiento que saque de la esfera de la independencia empresarial la determinación del precio o el proceso para su establecimiento. ${ }^{7}$

En este orden de ideas, cualquier acuerdo entre agentes del mercado que altere, modifique, influencie o de cualquier manera comprometa la independencia a la hora de fijar precios es ilegal. De hecho, para la SIC esto es tan claro que en una decisión anterior llegó a aseverar que la ley "prohíbe los acuerdos de precios sin distinguir entre verticales u horizontales" ${ }^{8}$, lo que creó toda una línea doctrinaria que hasta hace relativamente poco fue debidamente revisada por nuestra autoridad.

\section{La fijación vertical de precios}

A nivel de relaciones verticales, la fijación de precios tiene unas características y unos efectos muy distintos a la fijación horizontal -la que sucede entre competidores-. La SIC lo ha establecido de la siguiente manera:

Pues bien, como tales disposiciones [Artículo 1 de la Ley 155 de 1959 y Artículo 47 del Decreto 2153 de 1992] no diferencian entre acuerdos verticales y horizontales, la SIC ha considerado que para poder sancionar por acuerdos contrarios a la libre competencia es irrelevante que los mismos tengan una u otra naturaleza.

(...)

No obstante lo anterior, hoy en día, a partir de la experiencia extranjera y teniendo en cuenta los estudios económicos que se han hecho sobre este tema, la SIC considera necesario replantear el acercamiento hacia los acuerdos verticales de fijación de precios que sugiere de manera general la literalidad de la norma. Esto, teniendo en cuanta que la Autoridad de Competencia reconoce que las restricciones de esa naturaleza en determinadas ocasiones y bajo ciertos supuestos, pueden generar eficiencias deseables (...). ${ }^{9}$

El tema de la fijación vertical de precios es tanto apasionante como misterioso, y alcanza para innumerables artículos, ensayos y opiniones. La doctrina nacional cuenta con trabajos de muy alta calidad al respecto ${ }^{10}$. Para efectos del presente escrito, basta con resumir las conclusiones de la SIC frente a la fijación vertical de $\operatorname{precios}^{11}$ :

- Los precios máximos y sugeridos de reventa están permitidos puesto que no limitan que el agente aguas abajo establezca precios más bajos para competir en el mercado. 
- Los precios fijos y mínimos deben ser analizados caso por caso; aunque éstos tienen el potencial para limitar la competencia, también puede generar efectos procompetitivos. Por ello, se deben ponderar tanto los efectos negativos como positivos que se desprenden de la fijación de este tipo de precios, para determinar si, bajo las circunstancias propias del mercado, deben ser considerados anticompetitivos.

Por lo anterior, y con base en la doctrina actual de la SIC, el análisis de un acuerdo de precios depende del tipo de acuerdo -horizontal o vertical- que se investigue, no siendo los segundos per se ilegales.

\section{Las tarifas en las ERT}

Una de las innovaciones tecnológicas que más impacto ha tenido sobre los mercados son las aplicaciones móviles de transporte. Las mismas, como se desarrolla en detalle más adelante, han permitido superar altos costos de transacción para abrir la puerta a servicios que, sin la tecnología actual, eran imposibles de proveer. La Comisión Federal de Competencia Económica, autoridad de competencia de México, en una opinión ya famosa -y premiada por el Banco Mundial y la Red Internacional de Competencia ${ }^{12}$ - indicó:

Basándose en el desarrollo de tecnologías de teléfonos inteligentes y los sistemas de posicionamiento global, recientemente han surgido diversas empresas dedicadas a mediar el acuerdo entre usuarios y proveedores de servicios de transporte a través de aplicaciones en teléfonos móviles (...). Con el uso de este tipo de aplicaciones descargables en dispositivos móviles (plataformas), los usuarios demandan servicios de transporte de punto-a-punto; por otra parte, un grupo de conductores privados ofrece el servicio mediante el uso de la misma aplicación y de vehículos propios.

(...)

Estas nuevas plataformas construyen un nuevo producto en el mercado, ya que ofrecen al pasajero, además de movilidad, atributos nuevos y diferenciados en cuanto a (i) confiabilidad y seguridad personal, (ii) certidumbre en cuanto al cobro que se va a realizar y el método de pago, (iii) confort y conveniencia, (iv) búsqueda de tiempos de espera, e (v) información sobre el traslado. Adicionalmente, una característica particular de este servicio radica en las externalidades que se generan entre los usuarios y prestadores, pues a mayor cantidad de usuarios conectados mayor será la disposición de conductores a estar conectados, y viceversa. ${ }^{13}$

Las ERT, entonces, permiten superar fricciones existentes en el modelo de transporte, conectando usuarios con conductores ${ }^{14}$. Una de sus principales características es el establecimiento de una tarifa, del precio que debe pagar el usuario por ser transportado del origen a su destino. Sin embargo, esto elimina la capacidad del conductor de cobrar libremente el precio, y, potencialmente, limita el que los usuarios puedan acceder a precios más bajos para el mismo servicio. Entonces, ¿estamos ante un caso de fijación de precios? De ser así, ¿es horizontal o vertical? Y, por último, ¿puede decirse que esta fijación está justificada en los beneficios y eficiencias que genera?

Antes de entrar en materia, es importante anotar que la posible fijación de precios ya fue motivo de una acción de grupo (class action) en contra de una ERT en los Estados Unidos ${ }^{15}$. El demandante alegó que existía una fijación de precios pues la ERT limita la competencia entre los conductores al no permitirles negociar con los usuarios. La demanda señala que el acuerdo es horizontal al resultar en un acuerdo entre los competidores (conductores) ${ }^{16}$.

Sin embargo, el proceso no resultó en una decisión al respecto; el 5 de marzo de 2018, la corte confirmó su decisión de dar la razón al demandado y ordenar que el caso fuera decidido en un arbitramento. La base de esta decisión fue que los términos y condiciones, aceptados por el demandante, establecían que cualquier disputa debía ser llevada a dicho foro ${ }^{17}$.

Por otro lado, una denuncia básicamente idéntica fue decidida en noviembre de 2017 por la Comisión de Competencia de la India. La autoridad encontró, en ese caso, que las ERT denunciadas no habían violado las normas de competencia y que "los conductores pueden haber aceptado que los precios fueran fijados por el algoritmo (pero) esto no implica una colusión entre los conductores" ${ }^{18}$. 


\section{La naturaleza del posible acuerdo}

En primer lugar, vale la pena analizar cuál sería la naturaleza del posible acuerdo restrictivo. Es decir, determinar si existe una relación horizontal o vertical, como ha sido entendida por la ley y la doctrina de la SIC, o si, como se sugiere, estamos ante un caso particular que no cabe dentro de estas definiciones tradicionales.

\section{Acuerdo vertical}

Los acuerdos verticales son definidos por la SIC como "aquellos convenios entre agentes económicos que se encuentran en diferentes niveles de la cadena de producción (...)"19 y que están "destinadas a regular la interacción entre agentes económicos independientes situados en diferentes niveles de una cadena de producción" ${ }^{20}$. El término "cadena de producción", a pesar de ser el preferido en las definiciones anteriores, no tiene una definición legal. No obstante, la Circular Única de la SIC, en el Título VII sobre protección de la competencia, sí define un concepto análogo:

Se entiende por cadena de valor: el conjunto de actividades a partir de las cuales es posible generar un ordenamiento en el que el producto obtenido en una actividad resulta ser insumo para otra. De esta manera, cada actividad o eslabón le agrega sucesivamente valor al producto, desde su creación hasta que llega al consumidor final. ${ }^{21}$

Es decir que un acuerdo vertical existe cuando se presenta entre agentes que tienen una relación insumoproducto. Para tomar el ejemplo de la SIC, "el que surgiría entre un productor de maíz y un distribuidor mayorista de arepas"22.

En ese sentido, la fijación de una tarifa por parte de una ERT no parece ser un acuerdo vertical, pues difícilmente hay una relación insumo-producto entre la ERT y un conductor vinculado. Esto es, de la misma manera que el directorio telefónico de las páginas amarillas no provee un insumo a los anunciantes listados.

\section{Acuerdo horizontal}

Por otro lado, podría hablarse, como en los antecedentes internacionales antes referidos, de un acuerdo horizontal ${ }^{23}$ entre los conductores inscritos. Naturalmente, no puede hablarse de un acuerdo de esta naturaleza entre conductores y ERT, pues no son competidores, ni, tampoco, de un acuerdo directo entre los conductores, por lo que estaríamos ante los denominados acuerdos bub-and-spoke.

No obstante, como se pasa a explicar a continuación, la naturaleza de la relación entre ERT y conductores inscritos tampoco parece cubierta por las acepciones tradicionales de este tipo de acuerdos.

\section{Acuerdos hub-and-spoke}

La doctrina de los acuerdos hub-and-spoke ha sido desarrollada principalmente en los Estados Unidos. Su nombre es una referencia a las ruedas de una bicicleta, donde los competidores se hallan en el borde (rim) y se conectan a través de los radios (spokes) al centro de la llanta ( $h u b)$ :

En el derecho de la competencia, un acuerdo hub-and-spoke es un cartel en el que un agente (el hub) coordina la colusión (en el neumático o borde de la llanta) a través de agentes aguas arriba o aguas abajo (los radios) vía restricciones verticales. Estos acuerdos pueden ser ilegales bajo las normas de competencia si el acuerdo entre los radios (el borde) es per se anticompetitivo $(\ldots) \cdot{ }^{24}$ 
Estructurar un cartel es difícil, no sólo porque la coordinación implica unos costos significativamente altos -por ejemplo, identificar posibles miembros, intercambiar información o comunicarse de forma segura, monitorear el cumplimiento y castigar a quienes se desvíen y, por supuesto, la posible sanción a la que se está expuesto-, sino también porque hay un incentivo económico para que los miembros no cumplan con el acuerdo.

La estructura de hub-and-spoke reduce los costos de coordinación y monitoreo, haciendo factible la creación de un acuerdo que, en circunstancias normales, sería demasiado costoso ${ }^{25}$. El hub se encarga de reducir los costos de coordinación al imponer las condiciones que los competidores aguas abajo deben cumplir, tiene acceso a información completa y puede, incluso, ejecutar retaliaciones contra quienes no cumplan las condiciones impuestas.

No obstante, las restricciones verticales, a pesar de poder facilitar un acuerdo horizontal, tienen efectos procompetitivos, como se anticipó anteriormente. Por ello, los precedentes estadounidenses han indicado que la sola existencia de restricciones verticales y una conducta paralela en el plano horizontal no son suficientes para encontrar un acuerdo anticompetitivo ${ }^{26}$. Por el contrario, "debe haber evidencia directa o circunstancial que razonablemente pueda probar que [los competidores] tenían un compromiso consciente de actuar en un esquema diseñado con un propósito ilícito" 27.

De hecho, se ha llegado a afirmar que sólo hay un grupo de restricciones verticales aisladas y un paralelismo que no alcanza para encontrar un acuerdo horizontal sin el acuerdo entre los competidores. Aunque existe una corriente doctrinaria que aboga por una posible "rueda sin neumático" o "rueda sin borde" (rimless wheel), ésta aún no cuenta con apoyo ${ }^{28}$-al punto que un aparte muy famoso de una decisión en los Estados Unidos se pregunta: "¿qué sería de una rueda sin neumático?”29.

La SIC no ha sido ajena a los acuerdos facilitados o coordinados por terceros. Sin embargo, su análisis se ha centrado en el rol de las asociaciones y agremiaciones como foros de intercambio de información y coordinación entre competidores. $\mathrm{Al}$ respecto, ha indicado:

[S] e ha reconocido que las asociaciones son entidades que a pesar de que sus actividades en su gran mayoría pueden ser benéficas para un sector determinado de la economía, también tienen la potencialidad de convertirse en un centro idóneo para la realización de conductas anticompetitivas por parte de sus asociados. ${ }^{30}$

Así las cosas, las asociaciones son un ejemplo claro de posibles acuerdos en la modalidad hub-and-spoke pues reducen los costos propios de la cartelización al agrupar en un mismo foro a varios competidores y teniendo acceso a la información de cada uno.

\section{Las ERT como acuerdos hub-and-spoke}

Ahora bien, ¿es aplicable la teoría de los acuerdos hub-and-spoke a las ERT y la fijación de las tarifas? Aunque de entrada parecería que el esquema podría asimilarse, hay razones que indican lo contrario.

En primer lugar, como se indicó anteriormente, la relación entre las ERT y los conductores inscritos no es vertical bajo la definición de las normas colombianas de competencia. No podría hablarse estrictamente de "restricciones verticales" pues falta el elemento esencial en cuanto no hay una relación insumo-producto en una misma cadena de valor. En ese orden de ideas, no hay un agente aguas arriba que facilite un acuerdo aguas abajo.

Lo que hay son unos términos y condiciones de uso de un programa, aplicación o plataforma en los que se encuentra la fijación de una tarifa por parte de quien la opera; mas no una fijación de precios de reventa para un bien o servicio a un agente aguas abajo.

En segundo lugar, estaríamos ante una "rueda sin borde", pues no existe un acuerdo entre los conductores inscritos en la ERT para incumplir las normas de competencia. Esto es, no hay una intención de los 
conductores de acceder o inscribirse en la ERT para evitar competir. Caso contrario a lo que sucedió, por ejemplo, en la sanción a ACEMI y las EPS en 2011. En palabras de la propia SIC, "las EPS-C agremiadas acordaron, en el marco de ACEMI y con la promoción de esta asociación, lo siguiente (...)”31 . Nótese cómo la SIC expresamente resalta el acuerdo entre las EPS -entre los competidores- con la promoción del actor indirecto. Es decir, la conducta anticompetitiva fue el acuerdo entre los competidores, a nivel del borde o neumático de la rueda, y no sólo por la existencia de una comunicación o intercambio de información con el hub.

Tercero, los conductores inscritos en una ERT son competidores precisamente por inscribirse en la ERT, mas no eran competidores susceptibles de cartelización antes de inscribirse a la ERT. Es decir, no deja de haber competencia por las ERT, sino que hay competidores por las ERT.

La distinción es sutil, pero muy relevante. La idea fundamental es que se puede hablar de cartel huband-spoke cuando hay un grupo de competidores a los que les resulta muy costoso llegar a un acuerdo anticompetitivo.

Volviendo a la sanción de ACEMI, las EPS probablemente hubiesen encontrado muy costoso llegar al acuerdo sin la intervención de la asociación. El mercado estaba en alguna medida atomizado, la coordinación era compleja, el intercambio de información directo también era difícil. La asociación redujo esos costos al punto que permitió centralizar la información y proporcionar un foro de discusión y seguimiento del acuerdo.

Las ERT son un caso muy distinto. Antes de que un conductor particular se inscriba a la misma, no está en competencia con otros conductores particulares. Es solo al aceptar los términos y condiciones que el conductor entra al mercado. En ese sentido, no hay una posible cartelización facilitada por la ERT porque no hay un acuerdo preexistente que se haga menos costoso.

Esto va en línea con el punto anterior de la rueda sin borde: no hay coordinación entre los competidores, sino sólo unas relaciones contractuales -ni siquiera restricciones verticales propiamente entendidas- entre el operador de una plataforma y cada usuario. Con esto en mente, se entiende perfectamente la conclusión de la Comisión de Competencia de la India cuando indica que "los conductores pueden haber aceptado que los precios fueran fijados por el algoritmo (pero) esto no implica una colusión entre los conductores"32.

\section{Posibles efectos en el mercado}

La pregunta que surge es, entonces, ¿ bajo qué regla se debe analizar esta fijación de precios?

Como se indicó anteriormente, la relación entre la ERT y un conductor inscrito no es una relación horizontal -pues ciertamente no compiten ni se dedican a la misma actividad- ni hay evidencia de un acuerdo horizontal entre los conductores inscritos facilitado por la ERT. En ese sentido, no debería encontrarse la fijación como per se ilegal, ya que esto está reservado para los carteles tradicionales quienes manejan acuerdos horizontales de fijación de precios.

Asimismo, como se expuso anteriormente, tampoco existe una relación vertical en el sentido de las normas colombianas de competencia que requieren un vínculo insumo-producto.

Estamos, entonces, ante un caso distinto, un mercado bilateral (two-sided market):

Los bares y las compañías de citas en línea ofrecen servicios a hombres y mujeres que quieren conocerse. Las tarjetas de crédito permiten a los tarjetahabientes y comercios que las reciben realizar transacciones. Las plataformas de videojuegos, como PlayStation o Nintendo, ofrecen software que permiten a los desarrolladores crear juegos y una consola en la que los consumidores pueden jugarlos. En cada caso, la compañía provee una plataforma que permite a dos grupos de consumidores distintos pero relacionados generar un valor. ${ }^{33}$

Al ser una relación diferente a la relación unidireccional del productor-consumidor, las herramientas tradicionales de análisis del derecho de la competencia no aplican de la misma manera ${ }^{34}$. 
Por ello, entre una condena de ilegalidad per se que no examine los efectos de estas plataformas y una aproximación de ponderación de efectos positivos y negativos, resalto la segunda como la más adecuada. Ésta, en últimas, permite analizar el impacto real de la fijación de precios por parte de estas plataformas -en este caso particular, las ERT- y sus efectos tanto perjudiciales como beneficiosos.

\section{Restricción a la competencia en precios}

La restricción alegada tanto en la denuncia en la India como en la acción en la corte de Nueva York se basó en la imposibilidad que tienen los conductores inscritos a una ERT de fijar la tarifa que le cobran al usuario que los contacta a través de la plataforma. Estas tarifas son fijadas por un algoritmo que tiene en cuenta factores como el origen y el destino, la hora del día, la distancia y el tiempo estimado, entre otros. Así, independientemente del conductor contactado, el viaje solicitado por el usuario tendrá el mismo precio. Esto, en últimas, asegura que al usuario le sea indiferente el conductor con quien se contacte -en términos de precios, no en cuanto a su puntuación-.

Sin embargo, es posible que un conductor pueda cobrar un precio inferior al usuario que el calculado por el algoritmo de la aplicación. Por ejemplo, un conductor con un vehículo de mejor tecnología en consumo de gasolina (A) -o eléctrico- tendría un menor costo que un conductor con uno menos eficiente (B). Asumamos un viaje con un costo arrojado por la aplicación de $\$ 10$. La ERT cobra un porcentaje de $20 \%$, por lo que el conductor recibirá $\$ 8$. El gasto de gasolina del vehículo de $\mathrm{A}$ es de $\$ 3$, mientras que el de $\mathrm{B}$ de $\$ 4$. En ese sentido, la utilidad de $\mathrm{A}$ es de $\$ 5$, mientras que la de $\mathrm{B}$ es $\$ 4$ (asumiendo, para estos efectos, que no hay más costos relevantes). El conductor A, entonces, podría reducir la tarifa del viaje, hasta $\$ 8,80$, punto en el cual su utilidad se igualaría con la de B.

La diferencia en el precio del viaje -\$1,20-implicaría un beneficio para el usuario, quien pagaría un menor valor que el arrojado por el algoritmo de la ERT. Sin embargo, no se permite que los conductores negocien libremente los precios con los usuarios.

Al final, este ejemplo lleva a que los consumidores terminen pagando precios superiores que, en ciertos casos, estarían por encima del nivel que se vería en un mercado en competencia. El efecto es el de precios artificialmente altos; en términos económicos, muy similar a un cartel.

\section{Posibles efectos beneficiosos}

Por otro lado, la fijación de precios por parte de las ERT genera beneficios y eficiencias tanto para conductores inscritos como para usuarios, como se explica a continuación.

\section{Reducción de costos de transacción}

El primero de los beneficios de la fijación de precios por parte de las ERT viene de los costos asociados a la negociación individual entre usuarios y conductores inscritos. Es decir, si cada conductor pudiera establecer un precio diferente, el usuario se vería inmerso en un escenario de negociaciones costosas.

Asumamos que un usuario $\mathrm{X}$ necesita desplazarse y, para lograrlo, abre la ERT que tiene instalada en su teléfono inteligente. Esta ERT no fija los precios, por lo que cada conductor es libre de ofrecer el servicio al precio que quiera. Los conductores disponibles para X son A, B y C. A está dispuesto a ofrecer el servicio por no menos de $\$ 10$, B por no menos de $\$ 11$ y $\mathrm{C}$ por no menos de $\$ 12$. Estas diferencias de precios pueden corresponder a muchos factores: por ejemplo, el vehículo de A es más eficiente y consume menos gasolina, o A está más cerca a $\mathrm{X}$ por lo que su desplazamiento le implica menos costos. 
En este caso, la mejor alternativa para $\mathrm{X}$, en términos de precio, es A, quien podría cobrarle (hasta) $\$ 10$ por el trayecto. La pregunta, entonces, es ¿cómo logra $\mathrm{X}$ conectarse con $\mathrm{A}$ ?

Una primera alternativa es que $\mathrm{X}$, una vez ingrese la información del trayecto, pueda contactar a los conductores disponibles (sea a través de una llamada, mensaje de texto o instantáneo) y preguntar a cada uno cuánto sería el valor del trayecto. Supongamos que X llama primero a A, quien le dice que $\$ 10$. X tiene dos opciones: aceptar o contactar a otro conductor. Si acepta, habrá conseguido el mejor precio; sin embargo, no tiene cómo saberlo sin comparar con los otros conductores disponibles y podría haber alguno dispuesto a llevarlo por menos (pues X no tiene suficiente información). Si, por el contrario, contacta a B y luego a C, se dará cuenta que A es su mejor opción.

No obstante, la inmediatez del servicio -punto clave que se abordará más adelante- hace probable que para cuando X vuelva a contactar a A, éste ya haya aceptado un pedido de otro usuario. Así, el tiempo de búsqueda de $\mathrm{X}$ es un costo significativo: no aceptar inmediatamente puede resultar en no obtener el mejor precio disponible por parte de los oferentes.

Es posible pensar en mecanismos diferentes que alivien en algo este costo. La ERT puede limitar el número de conductores que pueden ser contactados por el usuario y no permitirles aparecer en las búsquedas de otros hasta que la actual se resuelva. En el ejemplo anterior, X solicita el servicio y la ERT sólo le entrega la información de A, B y C. Éstos no podrán aceptar otro servicio hasta tanto X no elija.

Aunque esto permitiría a X recibir el mejor valor, también genera problemas. Por una parte, ¿cuáles 3 conductores se eligen? Esto es, podría haber un conductor D dispuesto a cobrar $\$ 9$, pero por estar más lejos, o por ser menos activo en la ERT, o por su puntaje de satisfacción -o cualquier otro motivo- no es visible para X. Por otra parte, durante el tiempo que A, B y C están "congelados" puede haber otro usuario con un trayecto distinto para el cual $\mathrm{C}$ sea la mejor opción -porque está dispuesto a cobrarle menos que los demás conductoreso que sea la mejor opción para $\mathrm{C}$-porque, por ejemplo, está más cerca o conoce la ruta más eficiente para ese desplazamiento-. Sin embargo, la limitación artificial de la ERT no le permite contactarse con ese usuario.

Además, aunque este sistema premia al usuario, de alguna manera, es menos benévolo con los conductores inscritos: por cada usuario hay un conductor "ganador" y dos "perdedores". Éstos, a pesar de estar disponibles y dispuestos a prestar un servicio, se quedan inutilizados al menos hasta que la ERT los asigne a un nuevo usuario.

Otra alternativa es un mecanismo donde alguna de las partes -conductor o usuario- establezca un precio. De hecho, la aplicación InDriver utiliza un sistema donde el usuario fija el precio que está dispuesto a pagar.

Este es un sistema no sólo innovador sino muy interesante. Básicamente, el usuario ingresa los datos del trayecto (origen y destino) y el valor que está dispuesto a pagar. Los conductores inscritos pueden aceptar, rechazar, u ofrecer un precio superior. Así, eventualmente, el usuario aceptará una contraoferta -o aumentará lo que ofrece- hasta que sea atractivo para algún conductor.

No obstante, también puede tener algunos inconvenientes. Por un lado, asume que el usuario tiene información completa sobre los desplazamientos y los costos asociados a los mismos. Esto significa, que la experiencia del usuario es suficiente para determinar un valor adecuado para un desplazamiento específico, lo que puede que no suceda -sobre todo teniendo en cuenta que el usuario no conoce factores relevantes como, por ejemplo, el estado del tráfico al momento de solicitar el servicio-. Por el otro, puede que el usuario no sea inflexible con su oferta y termine ofreciendo un valor contraproducente. Por ejemplo, el usuario X puede ofrecer un valor de $\$ 10$ por su viaje, pero si supiera de las condiciones de alta demanda estaría dispuesto a ofrecer más. $\mathrm{O}$, por poner otro escenario, $\mathrm{X}$ debe esperar 15 minutos al conductor que aceptó su oferta, sin saber que uno que estaba a sólo 5 minutos de su ubicación habría aceptado $\$ 12$-lo que $\mathrm{X}$ hubiera estado dispuesto a pagar por llegar más rápido a su destino-.

Al final, aun cuando no es imposible imaginar sistemas alternativos a la fijación del precio por parte de la ERT, parece que sí puede ser el sistema más eficiente, precisamente, al homogeneizar el servicio ofrecido. Es decir que al consumidor le es indiferente quién es el conductor que presta el servicio. 
De hecho, la regulación de tarifas en el servicio de transporte público -tanto individual como colectivoparece buscar exactamente este resultado. Un usuario es indiferente al taxi o bus que lo transporta porque, independientemente de la identidad del conductor, el desplazamiento tiene el mismo costo. Una tarifa pública no regulada se enfrenta a los mismos problemas y costos antes mencionados: negociaciones particulares usuario-conductor, selección adversa e información asimétrica.

El hacer indiferente al usuario acerca de la identidad del conductor es clave para la eficiencia que logran las ERT. Esto elimina las negociaciones individuales, asigna a los conductores de manera casi inmediata, y reduce los costos de transacción y búsqueda usuarios inscritos de lado y lado.

\section{El sistema de calificación}

Ahora bien, otro de los beneficios que puede traer la fijación de precios por parte de las ERT es que, en principio, aumenta la transparencia del sistema de calificación de conductores y usuarios. Aun cuando se resaltó anteriormente que las ERT hacen indiferente a las partes frente a su contraparte en cuanto a los precios, la identidad sí juega un papel fundamental para las ERT: el sistema de calificación. Los conductores y usuarios que reciben bajas calificaciones son penalizados o hasta removidos de la plataforma.

Si los precios fueran libremente negociados, se podría amenazar la transparencia del sistema de calificación. Esto es, podría haber usuarios mal calificados por el hecho de ofrecer precios bajos -en opinión del conductor que lo califica-o conductores bien calificados sólo por el hecho de aceptar precios bajos -en opinión del usuario calificador-.

Esto afecta positivamente la calidad del servicio ofrecido, tanto por usuarios como por conductores. $\mathrm{Al}$ remover el precio como factor sujeto a calificación, los conductores y usuarios califican estándares como amabilidad, estado del vehículo, respeto de normas de tránsito, etc. Esto conlleva a que las partes busquen ofrecer una calidad alta a su contraparte, para ser bien calificados.

Sin embargo, si el precio se convierte en factor de calificación, la calidad se diluye. Habría conductores que prestan un servicio no óptimo (vehículos en mal estado o violación de las normas de tránsito) que serían calificados más alto por el hecho de aceptar precios bajos. Del mismo modo, habría usuarios que serían merecedores de bajas calificaciones con un puntaje alto por el hecho de ofrecer más por sus desplazamientos.

Aunque es perfectamente racional aceptar un servicio de calidad inferior a la óptima si el precio es suficientemente bajo ${ }^{35}$, el sistema de calificación cumple una tarea informativa. Un conductor puede no estar dispuesto a transportar a un usuario que tiene una baja calificación a pesar de estar dispuesto a pagarle más. Sin embargo, por la distorsión del sistema, puede que no le sea fácil, o del todo posible, identificarlo como tal.

En últimas, esta falta de transparencia del sistema de calificación puede redundar en, por un lado, una baja general en la calidad de los servicios, y, por el otro, en la no penalización de conductores y usuarios que de otro modo estarían sujetos a este tipo de sanciones.

\section{Incentivo a las tecnologías eficientes}

Como se indicó anteriormente, el costo más significativo para los conductores inscritos en una ERT es el combustible consumido por el vehículo. No sólo porque el precio de cada viaje debe poder cubrir el combustible consumido en el mismo, sino que debe también alcanzar a cubrir el utilizado en el trayecto hacia la ubicación origen del usuario.

Lógicamente, los conductores inscritos tienen un incentivo para reducir este costo lo máximo posible, pues entre menor sea el costo, mayor será la utilidad. En este orden de ideas, un vehículo más eficiente representa mayores ganancias para los conductores inscritos. 
Por supuesto, hay que tener en cuenta que la inversión en un vehículo nuevo -o más eficiente- también es un costo relevante. Es posible que un conductor actualmente tenga un vehículo menos eficiente que un vehículo nuevo, pero cuyo costo ya haya cubierto. Para él, puede ser posible mantener un margen más alto con un vehículo menos eficiente pues el ingreso adicional por el ahorro de gasolina no cubre lo que debe pagar para cubrir el costo del vehículo nuevo.

No obstante, sí existe un incentivo para los conductores inscritos para modernizar la tecnología de sus vehículos, sobre todo si los márgenes de utilidad son suficientemente buenos como para cubrir la inversión inicial en nuevos vehículos.

Esto no sólo significa una ventaja para los conductores en términos de utilidades, sino también en otras posibles externalidades positivas como la modernización del parque automotor, la reducción de emisiones dañinas y mayor seguridad en las vías.

\section{Conclusiones}

Así las cosas, parece que la fijación de precios por parte de las ERT sí tiene el potencial para generar una serie de beneficios y eficiencias que serían imposibles de lograr a través de un sistema de negociación privada o particular entre conductores y usuarios. El efecto más importante siendo la reducción de los costos de transacción.

Asimismo, es indiscutible que existen plataformas en otras industrias que sirven el mismo propósito acercar oferentes y demandantes- que no fijan los precios directamente, y son tanto exitosas como eficientes.

Sin embargo, el caso de las ERT es particular por dos razones. Por un lado, la inmediatez del servicio, pues es uno que se solicita y consume en un periodo muy corto de tiempo. Al ser inmediato, el usuario no dispone de un tiempo suficiente como para contactar a los múltiples conductores disponibles, comparar los precios ofrecidos por cada uno, y tomar la decisión que más le conviene. Como vimos antes, para cuando el usuario termine de contactar al último conductor disponible, su opción más favorable puede ya no estar disponible por haberse alejado del lugar donde se encuentra el usuario o por haber aceptado otro servicio, por ejemplo-. $\mathrm{Al}$ igual que sucede con el servicio de transporte público, homogeneizar el precio tiene un efecto claro en la eficiencia: la asignación usuario-conductor es inmediata y ahorra los costos de buscar -e incluso de no obtenerel mejor precio posible.

Un caso análogo de otro tipo de aplicación innovadora es el de Rappi, Globo o similares. La aplicación fija el precio del servicio, en lugar de que lo haga cada uno de las personas inscritas en la misma. Esto tiene la misma finalidad: hacer indiferente al usuario sobre quién estará encargado de realizar y entregar su pedido. No es difícil imaginar los altos y desgastantes costos de transacción de realizar un pedido y contactar a todos los "rappitenderos" disponibles para buscar quién ofrece el servicio al menor precio.

Por el otro, en el caso de las ERT es el oferente quien se desplaza, no el demandante. En el caso de los hoteles o pasajes aéreos, entre otros, es el consumidor quien va a desplazarse para utilizar el servicio. En ese sentido, el hotel puede fijar el precio de una noche o la aerolínea el precio del pasaje sin considerar costos adicionales. El conductor inscrito en una ERT no puede hacer esto, pues dentro de sus costos debe contar el desplazamiento, que varía dependiendo de la distancia al lugar de origen del viaje, su margen de utilidad, costos, etc. Este cálculo no sólo es difícil, sino que puede producir variaciones muy grandes entre ofertas.

Si bien pareciese existir un efecto anticompetitivo al no permitir que un conductor pueda cobrar un precio menor al indicado por la ERT, puede que no sea tan clara su existencia. Como se mencionó anteriormente, los conductores inscritos en una ERT son competidores por el hecho de estar inscritos; antes de eso, o una vez decidan abandonar la ERT, no están ejerciendo una actividad ni compitiendo. Por ello, asimilar la fijación de precios por la ERT a la fijación tradicional horizontal, vertical o hub-and-spoke no es adecuada. 
En cualquier caso, parecería ser que los beneficios de la fijación de los precios compensan la posible restricción. Si se toma como aplicable un análisis de efectos -similar al aplicable en casos de acuerdos verticales- es posible llegar a que la ponderación de efectos resulta en que los beneficios sí compensan el efecto anticompetitivo. En últimas, la fijación de la tarifa permite la asignación eficiente de los recursos, promueve un sistema de calificación más transparente e, incluso, puede implicar un incentivo a inversiones en tecnologías más eficientes.

Las aplicaciones tecnológicas son indudablemente cada vez más relevantes en el día a día de la economía. Irrupciones como las de las ERT no sólo son innovadoras, sino que llevan a reconsiderar muchos de los conceptos económicos tradicionales que dejan de ser directamente aplicables. Es ahora tarea de las autoridades el decidir cómo tratar estas innovaciones, sin ser tan estrictos como para comprometer la creación de nuevas tecnologías y las eficiencias que generan en el mercado.

\section{Referencias}

4 In re Musical Instrs. and Equip. Antitrust Litig. (Guitar Center), 798 F.3d 1186, 1194 (9th Cir. 2015). https://govshare.gov.il/sites/default/files/docs/In\%20re\%20Musical\%20Instruments\%20\%26\%20Equ ip.\%20Antitrust\%20Litig._\%2079.pdf

Ahmore Burger-Smidt \& Graeme Wickins, The Uber Price-fixing Ride: What are the Antitrust Co-ordinates? (2016). https://www.werksmans.com/wp-content/uploads/2016/08/061416-Uber-Pricing-Fixing.pdf

Alasdair Pal Martin Howell \& Christopher Cushing, India's competition commission rejects price-fixing allegations against Uber and Ola, Reuters, (7 de noviembre, 2018). https://www.reuters.com/article/us-uber-ola-antitrust /indias-competition-commission-rejects-price-fixing-allegations-against-uber-and-ola-idUSKCN1NC1BE

Alfonso Miranda Londoño \& Gabriel Ibarra Pardo, El estado actual de la práctica defijación unilateral deprecios (FUP) en Colombia, 12 Revista Derecho de la Competencia, n. ${ }^{\circ}$ 12, 289-349 (2016). https://centrocedec.files.wordpr ess.com/2010/06/9-estado.pdf

Barak Orbach, Hub-and-Spoke Conspiracies, The Antitrust Source (2016). https://www.americanbar.org/content/da $\mathrm{m} / \mathrm{aba} /$ publishing/antitrust_source/apr16_orbach_4_11f.authcheckdam.pdf

Circular Única [Superintendencia de Industria y Comercio]. 6 de agosto de 2001. D. O. 44511.

Comisión Nacional de Competencia Económica de los Estados Unidos Mexicanos, Comunicado de prensa: Premian la opinión de COFECE sobre Empresas de Redes de Transporte (28 de abril de 2016). https://www.cofece.mx /banco-mundial-premia-opinion-de-cofece-sobre-empresas-de-redes-de-transporte/

David S. Evans \& Richard Schmalensee, The Industrial Organization of Markets with Two-Sided Platforms, 3 Competition Policy International, n. ${ }^{\circ}$ 1, 150-179 (2007). https://www.law.berkeley.edu/wp-content/uploads/ 2015/04/Evans-Schmalensee-The-Industrial-Organization-of-Markets-with-Two-Sided-Platforms-2007.pdf

David S. Evans, Two-Sided Market Definition, ABA Section of Antitrust Law, Market Definition in Antitrust: Theory and Case Studies (2009). https://papers.ssrn.com/sol3/papers.cfm?abstract_id=1396751

Decreto 2153 de 1992 [Presidencia de la República de Colombia]. Por el cual se reestructura la Superintendencia de Industria y Comercio y se dictan otras disposiciones. 30 de diciembre de 1992. D. O. 40.704.

Jonathan Stempel, Uber wins U. S. court appeal to push price-fixing case to arbitration, Reuters, (17 de agosto, 2017). https://www.reuters.com/article/us-uber-decision/uber-wins-u-s-court-appeal-to-push-price-fixing-cas e-to-arbitration-idUSKCN1AX1MU

Margaret C. Levenstein \& Valerie Y. Suslow, What Determines Cartel Success?, 44 Journal of Economic Literature, n. $^{\circ}$ 1, 1-53 (2006). https://papers.ssrn.com/sol3/papers.cfm?abstract_id=299415

Meyerv. Kalanick, No.1:2015cv0979796 (31 de marzo, 2016). https://law.justia.com/cases/federal/district-courts/n ew-york/nysdce/1:2015cv09796/451250/37/

Monsanto Co. v. Spray-Rite Services Corp., 465 U.S. 752, 762 (20 de marzo, 1984). https://supreme.justia.com/cases /federal/us/465/752/ 
Pleno de la Comisión Federal de Competencia Económica, Opinión OPN-008-2015 (4 de junio, 2015). https://ww w.cofece.mx/cfcresoluciones/docs/Mercados\%20Regulados/V6/16/2042252.pdf

Resolución 17539 de 2002 [Superintendencia de Industria y Comercio]. Por la cual se resuelve unos recursos. 4 de junio de 2002. https://www.sic.gov.co/sites/default/files/estados/RESOLUCION_17539_DE_04_DE_JUN IO_DE_2002.pdf

Resolución 25402 de 2002 [Superintendencia de Industria y Comercio]. Por la cual se impone una sanción y se prohíbe el ejercicio de una conducta. 6 de agosto de 2002. http://normograma.info/sic/docs/r_siyc_25402_2002.htm

Resolución 2830 de 2004 [Superintendencia de Industria y Comercio]. 23 de febrero de 2004.

Resolución 29302 de 2000 [Superintendencia de Industria y Comercio]. Por la cual se impone una sanción y se ordena la terminación de una conducta. 2 de noviembre de 2000. https://www.sic.gov.co/recursos_user/documentos/normatividad/Publicaciones_Despacho_3/sicdecisi ones2/RESOLUCIONES_COMPETENCIA_ANOS/2000/RESOLUCION_29302_DE_02_DE_NOVI EMBRE_DE_2000.pdf

Resolución 30300 de 2016 [Superintendencia de Industria y Comercio]. Por la cual se imponen unas sanciones. 23 de mayo de 2016. http://suin-juriscol.gov.co/viewDocument.asp?ruta=Resolucion/30035622

Resolución 40598 de 2014 [Superintendencia de Industria y Comercio]. Por la cual se ordena el archivo de una averiguación preliminar. 27 de junio de 2014. http://normograma.info/sic/docs/r_siyc_40598_2014.htm

Resolución 46111 de 2011 [Superintendencia de Industria y Comercio]. Por la cual se imponen unas sanciones. 30 de agosto de 2011.https://www.sic.gov.co/recursos_user/documentos/normatividad/Publicaciones_Despacho_3 /sicdecisiones2/RESOLUCIONES_COMPETENCIA_ANOS/2011/RESOLUCION_46111_DE_30_DE _AGOSTO_DE_2011_SANCION_ACEMI.pdf

Resolución 56816 de 2014 [Superintendencia de Industria y Comercio]. Por la cual se imponen unas sanciones a AESA (ASOCIACIÓN DE EMPRESAS SOCIALES DEL ESTADO DE ANTIOQUIA) por violación a la prohibición general del artículo 1 de la Ley 155 de 1959 y la prohibición a las Asociaciones o Sociedades Científicas y de Profesionales o Auxiliares del artículo 4 del Decreto 1663 de 1994. 24 de septiembre de 2014. h ttp://normograma.info/sic/docs/r_siyc_56816_2014.htm

Resolución 6110 de 2000 [Superintendencia de Industria y Comercio]. Por la cual se resuelve un recurso. 27 de marzo de 2000. http://normograma.info/sic/docs/pdf/r_siyc_6110_2000.pdf

Resolución 6839 de 2009 [Superintendencia de Industria y Comercio]. Por la cual se imponen unas sanciones. 9 de febrero de 2009.

Resolución 7950 de 2002 [Superintendencia de Industria y Comercio]. Por la cual se impone una sanción y se prohíbe el ejercicio de una conducta. 15 de marzo de 2002. http://visordocs.sic.gov.co:8080/consultaDocs/actos_admi nistrativos.jsf?tipo_acto $=$ RE\&ano_acto $=2002 \&$ nume_acto $=7950$

Siddhartha Banerjee, Carlos Riquelme \& Ramesh Johari, Pricing in Ride-share Platforms: A Queueing-Theoretic Approach, Department of Management Science and Engineering, Stanford University (2016). http://ssrn.com /abstract $=2568258$

Superintendencia de Industria y Comercio, Preguntas frecuentes: Régimen de Protección de la Competencia (s. f.). http://www.sic.gov.co/sites/default/files/files/Nuestra_Entidad/Publicaciones/Preguntas_frecuentes_Reg imen_de_Proteccion_de_la_Competencia.pdf

\section{Notas}

\section{* Artículo de reflexión}

1 Presidencia de la República de Colombia. (30 de diciembre de 1992) Decreto 2153 de 1992 "Por el cual se reestructura la Superintendencia de Industria y Comercio y se dictan otras disposiciones". Diario Oficial No. 40.704 del 31 de diciembre de 1992. Artículo 47. 
2 Resolución 30300 de 2016 [Superintendencia de Industria y Comercio]. Por la cual se imponen unas sanciones. 23 de mayo de 2016.

3 Ver, entre otras, Resoluciones 6110 de 2000, 17539 de 2002 y 25402 de 2002 de la SIC.

4 Ver Resolución 7950 de 2002 [Superintendencia de Industria y Comercio]. Por la cual se impone una sanción y se prohíbe el ejercicio de una conducta. 15 de marzo de 2002.

5 Resolución 2830 de 2004 [Superintendencia de Industria y Comercio]. 23 de febrero de 2004.

6 Resolución 6839 de 2009 [Superintendencia de Industria y Comercio]. Por la cual se imponen unas sanciones. 9 de febrero de 2009.

7 Resolución 29302 de 2000 [Superintendencia de Industria y Comercio]. Por la cual se impone una sanción y se ordena la terminación de una conducta. 2 de noviembre de 2000.

8 Resolución 2830 de 2004 de la SIC.

9 Resolución 40598 de 2014 [Superintendencia de Industria y Comercio]. Por la cual se ordena el archivo de una averiguación preliminar. 27 de junio de 2014. Resaltado original de la resolución.

10 Ver, por ejemplo, Alfonso Miranda Londoño \& Gabriel Ibarra Pardo, El estado actual de la práctica de fijación unilateral de precios (FUP) en Colombia, 12 Revista Derecho de la Competencia, n. ${ }^{\circ}$ 12, 289-349 (2016).

11 Ver Resolución 40598 de 2014 de la SIC.

12 Comisión Nacional de Competencia Económica de los Estados Unidos Mexicanos, Comunicado de prensa: Premian la opinión de COFECE sobre Empresas de Redes de Transporte (28 de abril de 2016).

13 Pleno de la Comisión Federal de Competencia Económica, Opinión OPN-008-2015 (4 de junio, 2015).

14 Siddhartha Banerjee, Carlos Riquelme \& Ramesh Johari, Pricing in Ride-share Platforms: A Queueing-Theoretic Approach, Department of Management Science and Engineering, Stanford University (2016).

15 Meyer v. Kalanick, No.1:2015cv0979796 (31 de marzo, 2016).

16 Ahmore Burger-Smidt \& Graeme Wickins, The Uber Price-fixing Ride: What are the Antitrust Co-ordinates? (2016).

17 Jonathan Stempel, Uber wins U. S. court appeal to push price-fixing case to arbitration, Reuters, (17 de agosto, 2017).

18 Alasdair Pal Martin Howell \& Christopher Cushing, India's competition commission rejects price-fixing allegations against Uber and Ola, Reuters, (7 de noviembre, 2018).

19 Superintendencia de Industria y Comercio, Preguntas frecuentes: Régimen de Protección de la Competencia (s. f.).

20 Resolución 56816 de 2014 [Superintendencia de Industria y Comercio]. Por la cual se imponen unas sanciones a AESA (ASOCIACIÓN DE EMPRESAS SOCIALES DEL ESTADO DE ANTIOQUIA) por violación a la prohibición general del artículo 1 de la Ley 155 de 1959 y la prohibición a las Asociaciones o Sociedades Científicas y de Profesionales o Auxiliares del artículo 4 del Decreto 1663 de 1994. 24 de septiembre de 2014.

21 Numeral 2.1.1 del Título VII de la Circular Única de la SIC. Circular Única [Superintendencia de Industria y Comercio]. 6 de agosto de 2001. D. O. 44511.

22 Superintendencia de Industria y Comercio, Preguntas frecuentes: Régimen de Protección de la Competencia (s. f.).

23 "Se llama acuerdos horizontales a aquellos pactos entre empresas que proveen bienes o servicios similares o sustitutos en un único eslabón de la cadena de producción”. En Superintendencia de Industria y Comercio, Preguntas frecuentes: Régimen de Protección de la Competencia (s. f.).

24 Barak Orbach, Hub-and-Spoke Conspiracies, The Antitrust Source (2016). (Traducción libre)

25 Margaret C. Levenstein \& Valerie Y. Suslow, What Determines Cartel Success?, 44 Journal of Economic Literature, n. ${ }^{\circ}$, $1-53(2006)$. 
26 Barak Orbach, op. cit.

27 Monsanto Co. v. Spray-Rite Services Corp., 465 U.S. 752, 762 (20 de marzo, 1984).

28 Barak Orbach, op. cit.

29 "[F]or what is a wheel without a rim?" 4 In re Musical Instrs. and Equip. Antitrust Litig. (Guitar Center), 798 F.3d 1186, 1194 (9th Cir. 2015).

30 Resolución 46111 de 2011 [Superintendencia de Industria y Comercio]. Por la cual se imponen unas sanciones. 30 de agosto de 2011.

31 Resolución 46111 de 2011 de la SIC.

32 Alasdair Pal Martin Howell \& Christopher Cushing, op. cit.

33 David S. Evans, Two-Sided Market Definition, ABA Section of Antitrust Law, Market Definition In Antitrust: Theory And Case Studies (2009). (Traducción libre).

34 David S. Evans \& Richard Schmalensee, The Industrial Organization of Markets with Two-Sided Platforms, 3 Competition Policy International, n. $1,150-179$ (2007).

35 Por ejemplo, el caso típico de bienes sujetos a rebajas por defectos de fabricación, los cuales son adquiridos a precios reducidos a pesar de ser defectuosos.

\section{Licencia Creative Commons CC BY 4.0}

Cómo citar este artículo: Carlos Ignacio Arboleda Suárez, Sobre algoritmos y eficiencias: fijación de precios y empresas de redes de transporte, 69 Vniversitas (2020). https://doi.org/10.11144/Javeriana.vj69.saef 